\title{
Assessment of Left Ventricular Torsion in Hypertensive Patients
}

\author{
Walaa Farid, Amani Ouf, Rehab Yaseen \\ Cardiology Department, Faculty of Medicine, Menoufia University, Al Minufya, Egypt \\ Email:dr.cardio_amani@yahoo.com
}

How to cite this paper: Farid, W., Ouf, A. and Yaseen, R. (2019) Assessment of Left Ventricular Torsion in Hypertensive Patients. World Journal of Cardiovascular Diseases, 9, 109-121. https://doi.org/10.4236/wjcd.2019.92011

Received: August 1, 2018

Accepted: February 17, 2019

Published: February 20, 2019

Copyright () 2019 by author(s) and Scientific Research Publishing Inc. This work is licensed under the Creative Commons Attribution International License (CC BY 4.0).

http://creativecommons.org/licenses/by/4.0/

(c) (i) Open Access

\begin{abstract}
Background: Hypertension has important effects on left ventricle and its early detection helps to avoid the cardiac complications. LV Twist in systole helps in storing potential energy during diastole; LV untwist releases this energy. Speckle tracking echocardiography (STE) is angle-independent so it allows a more detailed evaluation of myocardial deformation. Objective: This work aimed to assess the left ventricular torsion by speckle tracking in hypertensive patients. Patients and Methods: The study was applied on 40 hypertensive patients and 20 age and sex matched control groups; all had speckle tracking echocardiography of the LV, and the basal and apical rotation were measured to assess the LV torsion. The 18 segments of LV strain were assessed and the net LV global longitudinal strain was calculated and demonstrated as bull's eye figure. Results: Hypertensive patients had higher mean values of both apical rotation and left ventricular twist than control group by a highly significant value $(9.76 \pm 4.98$ vs $4.03 \pm 2.15, \mathrm{P}$ value $<0.001)$ and $(15.25 \pm 4.10$ vs $9.90 \pm 1.47, \mathrm{P}$ value $<0.001)$ respectively. Hypertensive patients had a higher basal rotation than controls but did not reach a significant value $(-6.01 \pm 3.25$ vs $-5.52 \pm 2.63$, P value 0.558$)$. On the contrary, the global LV longitudinal strain was lower in hypertensive patients than control but did not reach a significant value $(-18.48 \pm 3.94$ VS $-19.48 \pm 3.57, \mathrm{P}$ value 0.341). Conclusion: Hypertension affects myocardial structure and its systolic and diastolic functions; the left ventricular twist is an essential component of the systolic function that increases in hypertension as an early compensation for the systolic impairment. The detection of these changes achieved accurately by $2-\mathrm{D}$ speckle tracking, can help in monitoring the treatment modalities of the patients for a better direction of treatment and thus, prevent further deterioration.
\end{abstract}

\section{Keywords}

Hypertension, Left Ventricular Torsion, Speckle Tracking Echocardiography 


\section{Introduction}

Hypertension affects $26 \%$ of the world's population and it is expected that its prevalence will be $29 \%$ by 2025 , mainly in economically developing countries [1]. Data from the Egyptian hypertension project (NPH) showed that hypertension is common in Egypt [2] [3].

$26 \%-30 \%$ of Egyptians have hypertension; more than $50 \%$ of individuals older than 60 years suffered from hypertension. At present if the same prevalence rate did not alter with population 110 millions in Egypt, there are approximately 29 million have hypertension. Only $38 \%$ of these patients are aware of having hypertension and only $24 \%$ are receiving treatment [4] [5] [6]. $15 \%$ $20 \%$ of patients with hypertension, develop LVH.

It was shown in many studies that a direct relation is found between LVH and the duration and stage of HTN [7]. LV Twist in systole helps in storing potential energy during diastole; LV untwist releases this energy. Twist helps LV ejection while untwist helps the left ventricular relaxation and filling. So rotation and torsion of the LV have an important role in cardiac mechanics [8].

Many investigators used different techniques to assess the cardiac twist and to detect its significance. Many of these techniques are invasive making them unsuitable for the human heart [9]. Assessment of LV systolic function is an important target of echocardiography. The parameter which is used for this purpose is LVEF. But it has several limitations, the most important: its inability to diagnose the early deterioration of contractile function so it is not helpful in diagnosing subclinical damage of the myocardium in a variety of clinical conditions and thus may have a major role in therapeutic and prognostic implications.

Strain imaging or "myocardial deformation imaging", helps in direct quantification of the degree of myocardial contraction thus overcoming the limitations of LVEF [10]. Speckle tracking echocardiography (STE) is a technique based on gray-scale, which is angle-independent so it allows a more detailed evaluation of myocardial deformation. The echocardiographic gray-scale image is composed of many bright speckles which resulted from scattering of the ultrasound beam by the tissue. These speckles are identified and tracked frame-by-frame by the software of STE using a sum of the absolute differences algorithm. From this data, the software automatically resolves the magnitude of myocardial deformation in different directions and generates the curves of strain [11] [12] [13].

Rotation is a rotatory motion around the center of the mass in the short axis image of LV [14]. When viewed from the apex, rotation in anticlockwise direction is expressed with positive values while rotation in clockwise direction with negative values. Normally during systole; the base of the heart rotates clockwise while the apex rotates anticlockwise so a wringing motion is produced. Torsion is measured as base to apex gradient in rotation angle along the left ventricular long axis, expressed in degrees per centimeters [15]. This work aimed to assess the left ventricular torsion by speckle tracking in hypertensive patients. 


\section{Patients and Methods}

This is a prospective study carried on 40 hypertensive patients recruited from Menoufia University hospital and 20 apparently healthy, age and sex matched volunteers as control group, after obtaining written consent, and approval of Ethic Committee of Menoufia University.

\subsection{Inclusion Criteria}

- Hypertension diagnosed by having one or more of these criteria based on ESH/ESC guidelines for diagnosis of hypertension: SBP $\geq 140 \mathrm{mmHg}$ and/or $\mathrm{DBP} \geq 90 \mathrm{mmHg}$ on two or more hospital visits at one-week interval, history of hypertension or maintenance on antihypertensive medications [16].

- Normal LV systolic function (ejection fraction > 50\%).

\subsection{Exclusion Criteria}

Patients with any of the following were excluded: LV systolic dysfunction $(\mathrm{EF}<$ $50 \%)$, diabetes mellitus [17], significant valvular disease, atrial fibrillation, ischemic heart disease, pericardial disease, congenital cardiac disease, renal or hepatic dysfunction, metabolic or endocrinal disorders, bad echogenic window.

\subsection{Methods}

All the study population subjected to the following: Full history taking, clinical examination, standard 12 leads ECG.

\subsection{Echocardiography}

Echocardiographic examination was done using Vivid 9, General Electric Healthcare (GE Vingmed, Norway) equipped with a harmonic M5S variable-frequency $(1.7-4 \mathrm{MHz})$ phased-array transducer. While the patient in the left lateral position recommended by the American Society of Echocardiography [18] and connected to single lead ECG.

\section{1) Conventional echocardiography}

- M-Mode: for measurement of wall thickness, dimensions, ejection fraction, LV mass, LV mass index and relative Wall thickness.

LV mass was calculated using the Devereux formula [19]

$\mathrm{LV}$ mass $=0.8\left(1.04\left(\left[\right.\right.\right.$ LVEDD + PWTD + IVSTD $\left.\left.^{3}-[\text { LVEDD }]^{3}\right)\right)+0.6 \mathrm{~g}$.

LV mass index $=$ LV mass/body surface area

The formula used for calculating RWT [20]: RWT $=2 \times$ PWD/LVEDD

- Doppler flow: The mitral inflow velocities were recorded and the following velocities were measured: peak velocity of early diastolic wave velocity (E), late diastolic wave velocity $(\mathrm{A})$ and $\mathrm{E} / \mathrm{A}$ ratio.

\section{2) Doppler tissue imaging}

A 1.5-mm sample volume was placed at the lateral mitral of the mitral annulus in apical 4-chamber view. Analysis was performed for the measurement of early diastolic wave velocity $\left(e^{\prime}\right)$ and $E / e^{\prime}$ ratio. 


\section{3) 2D speckle tracking echocardiography}

a) Measurement of global longitudinal strain of the LV

Three LV apical long axis views, apical four-chamber, two-chambers, and apical three chambers views were acquired; these views were taken at a frame rate ranging $59-82$ frame/s and stored digitally on hard disk for off-line analysis.

Mitral and aortic flow velocities were recorded using pulsed-wave Doppler to measure the timing of cardiac events. The endocardial border of LV at end-systolic frame was traced manually by strain software. Based on this line, the computer automatically created a region of interest (ROI) including the entire transmural wall, and the software selected suitable natural acoustic markers (speckles) moving with the tissue for tracking [13].

The software algorithm then tracks the speckle frame-by-frame by using the sum of absolute difference algorithm. At the end, the segments of good tracking quality are tracked and accepted automatically while poorly tracked segments are rejected by the software.

Automated frame-by-frame tracking of these markers during the cardiac cycle with the use of a dedicated software package yielded a measure of 2D LV strain and strain rate at any point of the myocardium. Offline analysis was done on a computer (PC) workstation using custom analysis software (Echopac PC, version 1.8.1 X GE Healthcare).

The 6 walls of the LV were automatically segmented into 18 equidistant segments model in a "bull's eye" plot using the software algorithm and each segment was individually analyzed to assess the global longitudinal LV strain [13] (Figure 1).

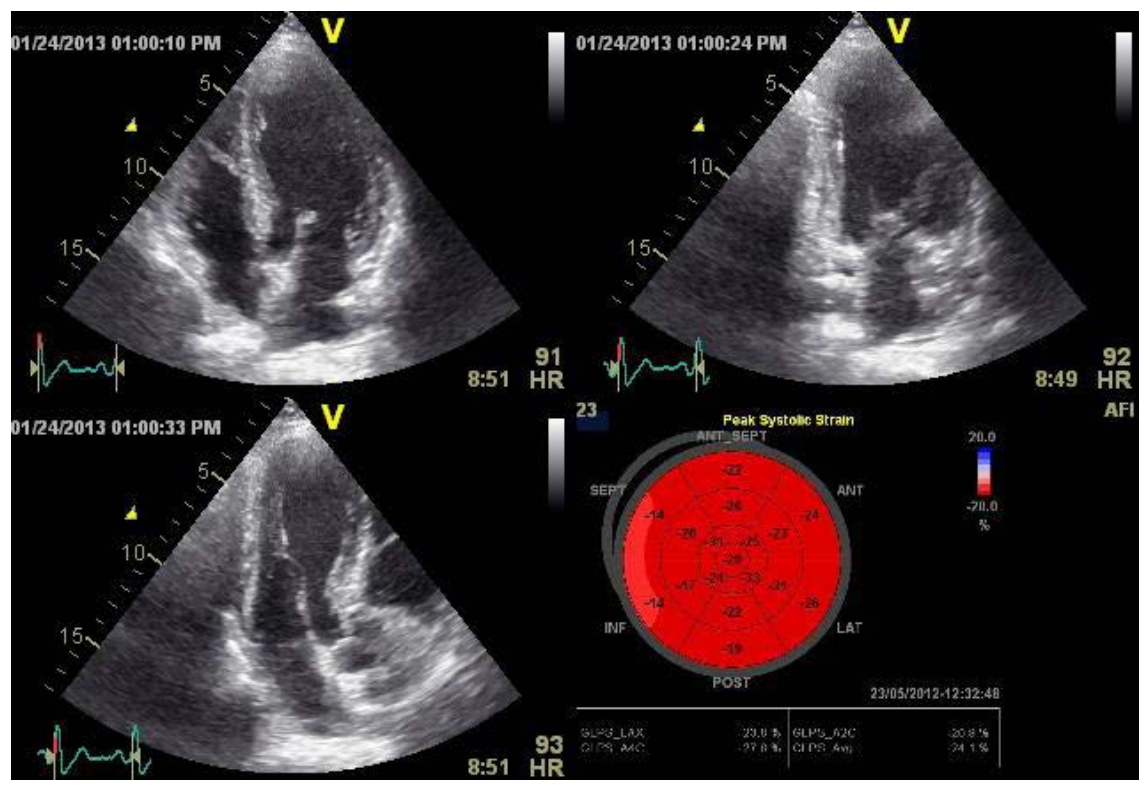

Figure 1. LV global longitudinal strain measured in apical long axis view, apical two chamber view and apical chamber view and bull's eye showing peak systolic strain of different segments of LV. 


\section{b) Measurement of LV twist}

Short axis view at the level of mitral valve was obtained by tilting the probe slightly upward until we got the characteristic fish mouth appearance of the mitral valve. Apical short axis view was obtained by tilting the probe more upward until we got a cross section of the LV apex. Speckle-tracking imaging analysis was performed using the available software (Echo PAC BT 12, GE-Vingmed; Norway).

The endocardial border of the LV was manually traced and then the software automatically generated a second, larger, concentric tracing at the epicardium so that all the LV myocardium became included. Then, the software automatically transformed each LV view into 6 equal segments and performed the speckle-tracking on a frame-to-frame basis [15].

Using the average of LV rotations from the 6 segments, we measured the basal and apical rotations taking into consideration to measure the mean rotation at aortic valve closure. The apical rotation was expressed in positive values, while the basal rotation in negative values. LV twist equals the apical rotation minus the basal rotation. Rotation and twist are expressed in degrees [15].

The pulsed wave Doppler tracing from the LV outflow tract was used to identify the timing of aortic valve opening and closure that marks systole (Figures 2-4).

\subsection{Statistical Analysis}

Data was statistically analyzed by using the well-known program statistical package for social science (SPSS) version 22 (Armonk, NY: IBM Corp, 2013)

Two types of statistics:

Descriptive statistics: e.g. percentage (\%), mean (x) and standard deviation (SD).

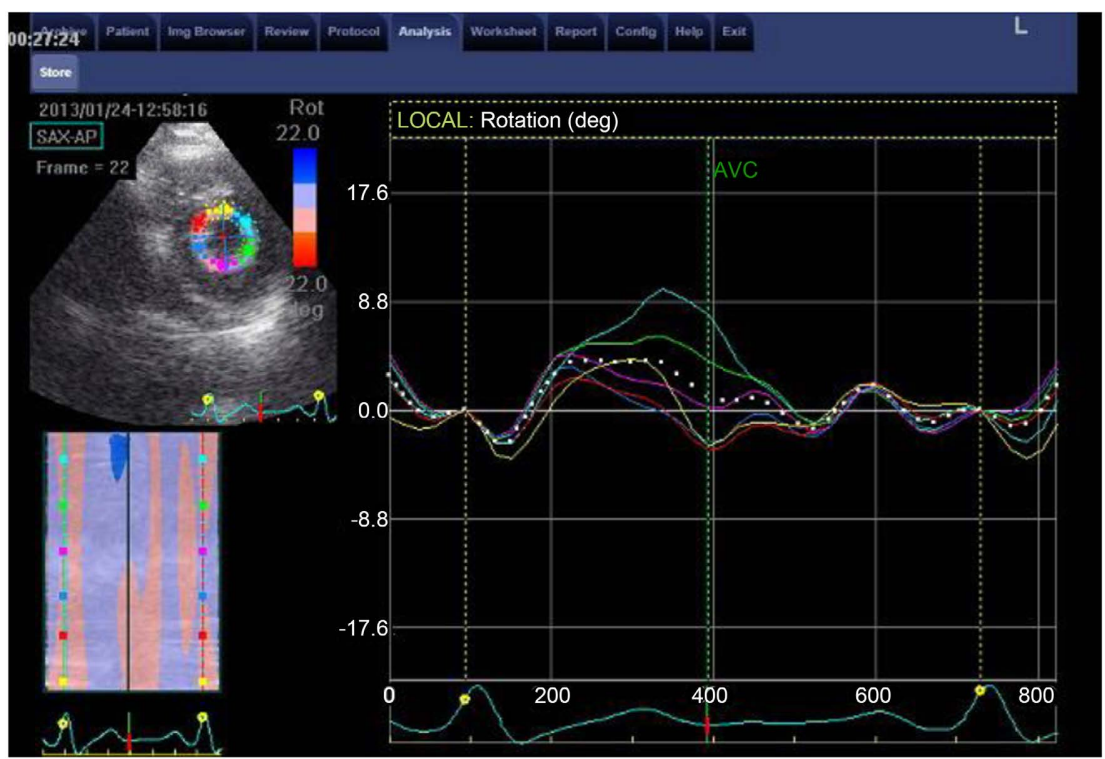

Figure 2. Apical rotation of LV measured at short axis view at apical level. 


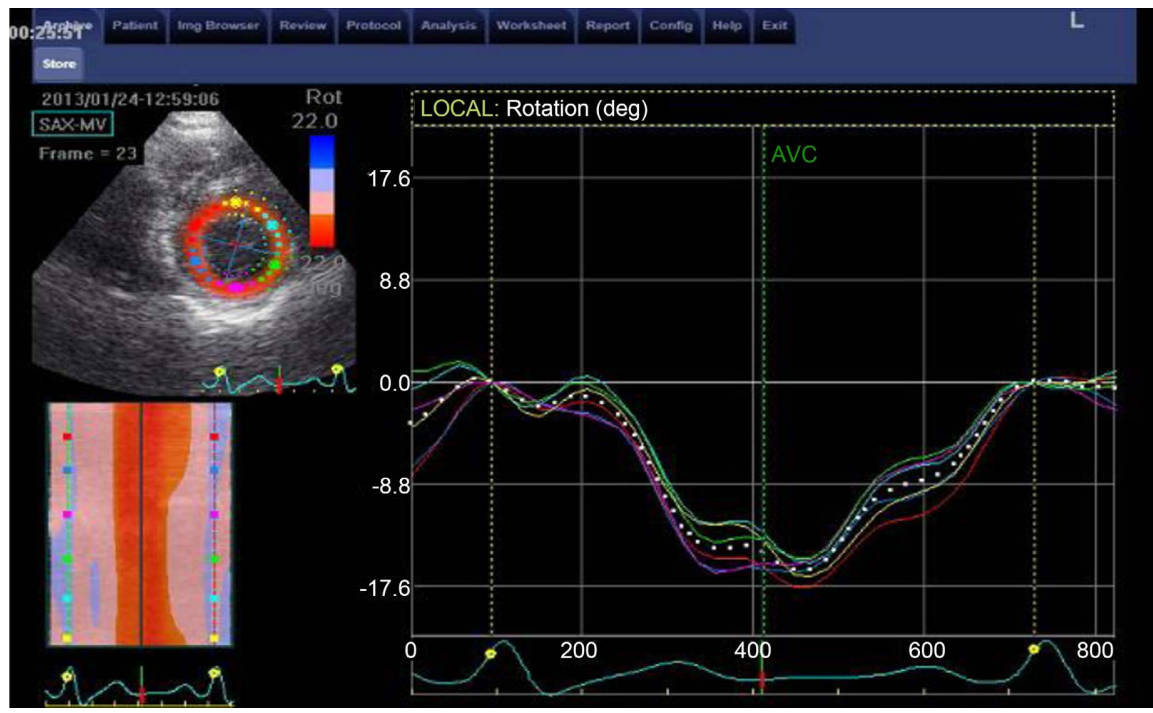

Figure 3. Basal rotation of LV measured at short axis view at mitral valve level.

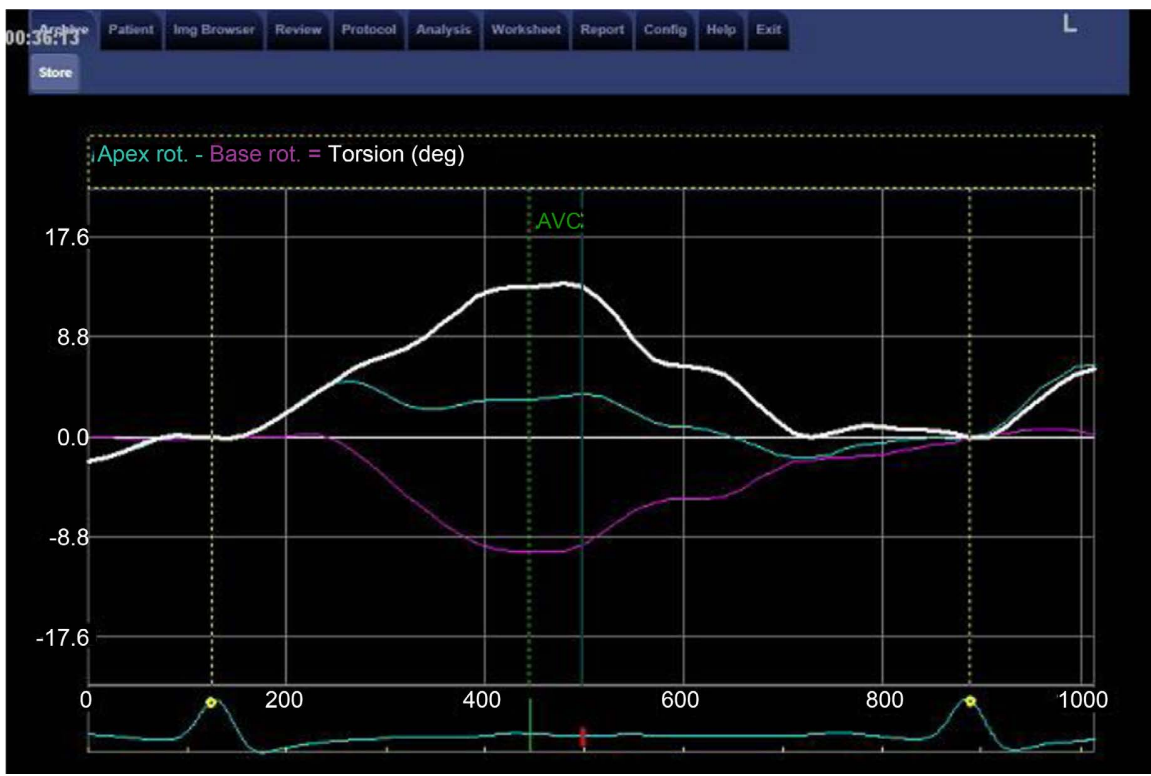

Figure 4. Measurement of LV torsion (LV torsion = apical rotation - basal rotation).

\section{Analytic statistics: e.g.}

- Chi-square test $\left(\chi^{2}\right)$ was used to study association between two qualitative variables.

- Students t-test is a test of significance used for comparison between two groups having quantitative variables.

- A p value was considered statistically significant below 0.05 .

\section{Results}

Forty hypertensive patients and 20 age and sex matched healthy controls were enrolled in this study.

There was no statistical significant difference between hypertensive patients 
and controls regarding age and sex ( $\mathrm{P}$ values $>0.05)$ (Table 1$)$.

We found that the interventricular septal thickness in diastole and the left ventricular posterior wall thickness in diastole were higher in the studied patients than the controls by highly significant values ( $\mathrm{P}$ value for both $<0.001$ ). Moreover left ventricular mass, LV mass index and relative wall thickness were higher in the studied patients than the controls by highly significant values $(P$ value $<0.001)$. Ejection fraction was higher significantly in the studied patients than controls (P value < 0.05) (Table 2).

When we studied the diastolic function we found that, A wave (late diastolic filling velocity of mitral flow) was higher in the studied patients than the controls

Table 1. Demographic data of the studied groups.

\begin{tabular}{ccccc}
\hline Demographic data & $\begin{array}{c}\text { Patients } \\
(\mathrm{n}=40) \\
\text { Mean } \pm \text { SD }\end{array}$ & $\begin{array}{c}\text { Controls } \\
(\mathrm{n}=20) \\
\text { Mean } \pm \mathrm{SD}\end{array}$ & $\begin{array}{c}\text { Test of } \\
\text { significance }\end{array}$ & P-value \\
\hline Age (years) & $54.00 \pm 9.51$ & $50.60 \pm 11.09$ & $\mathrm{t}=1.23$ & 0.222 \\
Sex: [No (\%)] & $14(35.0)$ & $\begin{array}{c}8(40.0) \\
\text { Males }\end{array}$ & $\chi^{2}=0.14$ & 0.705 \\
Females & $26(65.0)$ & $12(60.0)$ & & \\
\hline
\end{tabular}

Table 2. Comparison of conventional echocardiographic parameters in the studied groups.

\begin{tabular}{ccccc}
\hline Echocardiography parameters & $\begin{array}{c}\text { Patients } \\
(\mathrm{n}=40) \\
\text { Mean } \pm \mathrm{SD}\end{array}$ & $\begin{array}{c}\text { Controls } \\
(\mathrm{n}=20) \\
\text { Mean } \pm \mathrm{SD}\end{array}$ & t-test & P-value \\
\hline LVM $(\mathrm{g})$ & $205.25 \pm 42.86$ & $114.20 \pm 22.08$ & 8.90 & $<0.001^{*}$ \\
LVMI (g/m $)$ & $106.80 \pm 25.40$ & $59.60 \pm 10.35$ & 7.96 & $<0.001^{*}$ \\
RWT & $0.52 \pm 0.10$ & $0.33 \pm 0.05$ & 8.05 & $<0.001^{*}$ \\
LA (mm) & $36.83 \pm 5.63$ & $32.40 \pm 3.93$ & 3.15 & $0.003^{*}$ \\
AO (mm) & $31.08 \pm 4.35$ & $29.90 \pm 4.71$ & 0.96 & 0.341 \\
E (cm/s) & $66.08 \pm 16.99$ & $68.50 \pm 16.96$ & 0.52 & 0.60 \\
A (cm/s) & $77.15 \pm 16.06$ & $63.70 \pm 18.78$ & 2.89 & $0.005^{*}$ \\
E/A & $0.88 \pm 0.31$ & $1.06 \pm 0.24$ & 2.22 & $0.030^{*}$ \\
IVSD (mm) & $11.88 \pm 1.57$ & $7.60 \pm 0.94$ & 11.18 & $<0.001^{*}$ \\
LVPWD (mm) & $11.85 \pm 1.56$ & $7.80 \pm 0.77$ & 10.92 & $<0.001^{*}$ \\
LVEDD (mm) & $46.15 \pm 4.76$ & $46.70 \pm 4.23$ & 0.437 & 0.664 \\
LVESD (mm) & $28.43 \pm 3.48$ & $29.70 \pm 3.47$ & 0.13 & 0.185 \\
EF & $67.68 \pm 4.50$ & $64.60 \pm 3.70$ & 2.64 & $0.011^{*}$ \\
e' (cm/s) & $7.90 \pm 2.85$ & $10.10 \pm 1.68$ & 3.18 & $0.002^{*}$ \\
E/e' & $8.74 \pm 1.92$ & $6.59 \pm 1.34$ & 4.47 & $<0.001^{*}$ \\
\hline
\end{tabular}

${ }^{*}$ LVM: left ventricular mass, LVMI: left ventricular mass index, RWT: relative wall thickness. LA: left atrium, AO: aorta, E: early mitral filling wave, A: late mitral filling wave. IVSD: interventricular septum in diastole, LVPWD: left ventricular posterior wall in diastole, LVEDD: left ventricular end diastolic diameter, LVESD: left ventricular end systolic diameter, EF: ejection fraction. e': early diastolic myocardial velocity. 
by a significant value ( $\mathrm{P}$ value $<0.05)$. Also, E/e' (ratio between early diastolic filling velocity of mitral flow and the early diastolic myocardial velocity) was higher in the studied patients than the controls by a highly significant value $(\mathrm{P}$ value $<0.001)$, while, E wave was lower in hypertensive group than controls but didn't reach the significant value $(\mathrm{P}$ value $>0.05)$. On the other hand, the E/A ratio was lower in the studied patients than the controls by a significant value $(\mathrm{P}$ value < 0.05) (Table 2).

The left atrium diameter was significantly larger in the studied patients than the controls ( $\mathrm{P}$ value $<0.05)$ as shown in Table 2.

By performing speckle tracking on basal and apical levels of LV to assess their rotation degrees and the LV twist (torsion) we found that the apical rotation and the LV twist were higher in the studied patients than the controls by highly significant values $(\mathrm{P}$ values for both were $<0.001)$. While, basal rotation was higher in the studied patients than the controls but did not reach a significant level ( $P$ value $>0.05)$ (Table 3$)$.

By assessing the global LV longitudinal strain, we found that it was lower in the studied patients than the controls but didn't reach the significant value (P-value > 0.05) (Table 4).

\section{Discussion}

Although hypertension may be asymptomatic disease for a long time; it affects the heart. Its complications can occur even with hypertensive patients on medications, as a large percent of them are not controlled. According to this, the early detection of cardiac changes of hypertension especially changes in LV torsion is mandatory for prevention of more deterioration in both diastolic and systolic functions and also it helps in assessment of the efficacy of treatment [21].

Speckle tracking echocardiography measures different parameters of myocardial deformation, thus, provides information that cannot be detected by any of

Table 3. Comparison of left ventricular twist in the studied groups.

\begin{tabular}{ccccc}
\hline STE parameters & $\begin{array}{c}\text { Patients } \\
(\mathrm{n}=40) \\
\text { Mean } \pm \mathrm{SD}\end{array}$ & $\begin{array}{c}\text { Controls } \\
(\mathrm{n}=20) \\
\text { Mean } \pm \mathrm{SD}\end{array}$ & t-test & P-value \\
\hline Apex rotation & $9.76 \pm 4.98$ & $4.03 \pm 2.15$ & 4.91 & $<0.001^{*}$ \\
Base rotation & $-6.01 \pm 3.25$ & $-5.52 \pm 2.63$ & 0.59 & 0.558 \\
Twist & $15.25 \pm 4.10$ & $9.90 \pm 1.47$ & 5.63 & $<0.001^{*}$ \\
\hline
\end{tabular}

STE: speckle tracking echocardiography.

Table 4. Comparison of left ventricular global longitudinal strain in the studied groups.

\begin{tabular}{ccccc}
\hline STE parameters & $\begin{array}{c}\text { Patients } \\
(\mathrm{n}=40) \\
\text { Mean } \pm \mathrm{SD}\end{array}$ & $\begin{array}{c}\text { Controls } \\
(\mathrm{n}=20) \\
\text { Mean } \pm \mathrm{SD}\end{array}$ & t-test & P-value \\
\hline left ventricular global strain & $-18.48 \pm 3.94$ & $-19.48 \pm 3.57$ & 0.96 & 0.341 \\
\hline
\end{tabular}

*STE: speckle tracking echocardiography. 
the commonly used echocardiographic parameters. It assesses global and regional cardiac functions accurately by tracking ultrasound speckles in the myocardium on frame-by-frame basis [22].

We studied the effect of hypertension on LV twist and the global LV longitudinal strain to detect its early impact on the LV myocardial systolic function. Our study found that there was higher twist and apical rotation values in the studied patients than the controls by a highly significant value ( $\mathrm{P}$ value $<0.001$ for both) while basal rotation was higher in the studied patients than the controls but, did not reach a significant level ( $P$ value $>0.05$ ).

Fibrosis with increased amount of collagen fiber and fiber rearrangement in the subendocardial layer are the early pathological changes in HTN [23]. These changes lead to impairment of subendocardial contraction and decrease the opposing force to the counterclockwise rotation of the subepicardium. Consequently, the apical counterclockwise rotation increases [24].

This went in harmony with the findings of Cameli et al. [25] who found that, LV twist angle was increased in hypertensive patients compared to healthy controls $(\mathrm{P}$ value $<0.001)$.

In the early stages of hypertension; the increased LV twist seems to be a compensatory mechanism while in the advanced stages of hypertension; this hyper-torsion is lost. Similarly, Han et al. [26] found that both apical and basal rotations increased significantly in hypertensive group than control group; subsequently LV twist increased significantly (P value $<0.05$ ). The significant increase in basal rotation in hypertensive group may be attributed to larger number of patients in their study.

In our study, the global longitudinal strain of the LV was lower than in the studied patients control group but did not reach a significant value ( $\mathrm{P}$ value $>$ $0.05)$.

The global longitudinal strain of the LV is one of the parameters that assess systolic function and detects its early impairment before affection of ejection fraction [27]. Other studies with larger sample volume demonstrated a significant reduction in global LV longitudinal strain in hypertensive group than control group [28]. Sofi Taleb et al. studied longitudinal LV global strain in 200 hypertensive patients, and found that it was reduced in hypertensive patients $(<-17 \%)$. In univariate analysis, a reduced global longitudinal strain of LV was associated with HTN duration $>10$ years $(\mathrm{P}$ value $<0.005)$ also, reduced in uncontrolled HTN (P value < 0.0001) [28].

Similarly Walid Abdo et al. [29] found that global LV longitudinal strain reduced significantly in hypertensive group with LVH compared to control group $(\mathrm{P}$ value $<0.05)$ and compared to hypertensive group without LVH (P value < 0.05).

In our study left ventricular mass, LV mass index and relative wall thickness were higher in the studied patients than the controls by highly significant values (P value $<0.001$ ) due to the effect of hypertension on the LV geometry. Hyper- 
trophy of LV is a common response to increased and prolonged afterload [30].

Our results were concordant with Gonçalvesa et al. [30] who found that hypertensive patients had greater LV mass and greater relative wall thickness than the control group ( $\mathrm{P}$ value for both are $<0.001$ ).

Similarly Imbalzano et al. [31] found that LV wall thickness, LV mass index and relative wall thickness were significantly increased in both non-LVH and LVH hypertensive patients compared to the controls ( $\mathrm{P}$ value $<0.001$ ).

Our study showed that LA was significantly dilated in the studied patients than the controls $(\mathrm{P}$ value $<0.05)$. These results are concordant with the results of Cantoni et al. [32] who found that left atrium diameter is larger in hypertensive group than control group (P value $<0.05$ ). Also Haung et al. [33] who found that LA diameter is larger in hypertensive group either with or without LVH than controls (P value $<0.05$ ).

By analysis of mitral flow velocities we found that the late mitral A wave was higher in the studied patients than the controls by a significant value ( $\mathrm{P}$ value $<$ 0.005), and the E/A was lower in the studied patients than the controls by a significant value (P value $<0.05$ ). LV compliance is affected in hypertension due to the LV hypertrophy and subsequent ischemia of subendocardial layer so the developed stiffness is worsened by fibrosis, this causes an increase of LA pressure against the stiff LV eventually the E/A ratio decreases [33].

These results are concordant with Han et al. [26] who found that A wave was higher in hypertensive group than controls by a highly significant value ( $\mathrm{P}$ value $<0.001$ ) while $\mathrm{E}$ wave was lower in hypertensive group than controls by a significant value ( $\mathrm{P}$ value $<0.05)$. Moreover $\mathrm{E} / \mathrm{A}$ is lower in hypertensive group than controls by a highly significant value ( $\mathrm{P}$ value $<0.001$ ).

Also, Fangfang et al. [34] found that patients with essential hypertension had lower $\mathrm{E} / \mathrm{A}$ ratio compared to control $(\mathrm{P}$ value $<0.05$ ). Our results also went on harmony with Haung et al. [33] who found that $\mathrm{E}$ wave and E/A ratio was lower in hypertensive group either with or without LVH than control group ( $\mathrm{P}$ value $<$ $0.05)$, on the contrary the A wave was higher in hypertensive group than controls $(\mathrm{P}$ value $<0.05)$.

In our study we found that the hypertensive group had a highly significant higher $\mathrm{E} / \mathrm{e}^{\prime}$ ratio than controls ( $\mathrm{P}$ value $<0.001$ ). The results were in agreement with Fangfang et al. [34] who found that hypertensive patients had lower e' and higher $\mathrm{E} / \mathrm{e}^{\prime}$ ratio than the healthy subjects $(\mathrm{P}<0.05$ for both). Additionally Božidarka et al. [35] found that many hypertensive patients had E/e' ratio $>8$ (74.6\% of 449 patients).

Early mitral flow velocity (E) correlates with filling pressure but the abnormalities are non-linear as its measurement is influenced by both relaxation of the myocardium and filling pressure. Mitral E velocity is proportionate to the ratio between LA pressure and the relaxation time constant (tau) whereas e' is inversely proportionate to tau only, leading the ratio $\mathrm{E} / \mathrm{e}$ ' to be proportionate to LA pressure [35]. 


\section{Limitations}

The studied sample was small. Hypertensive patients had different onset, severity, and treatment modalities of their disease. The effects of age and obesity on LV twist were not excluded. Additionally there was no follow up of the patients to determine the improvement of LV torsion with better control of the hypertension.

\section{Conclusion}

Hypertension affects myocardial structure and its systolic and diastolic functions; the left ventricular twist is an essential component of the systolic function that increases in hypertension as an early compensation for the systolic impairment. The detection of these changes achieved accurately by 2 -D speckle tracking, can help in monitoring the treatment modalities of the patients for a better direction of treatment and thus, prevent further deterioration.

\section{Conflicts of Interest}

The authors declare no conflicts of interest regarding the publication of this paper.

\section{References}

[1] Kearney, P.M., Whelton, M., Reynolds, K., et al. (2005) Global Burden of Hypertension: Analysis of Worldwide Data. Lancet, 365, 217-223. https://doi.org/10.1016/S0140-6736(05)70151-3

[2] Ibrhim, M.M. and Albertino, T. (2012) Hypertension in Developed Countries. Lancet, 380, 611-619. https://doi.org/10.1016/S0140-6736(12)60861-7

[3] Ibrahim, M.M. (1999) Epidemiology of Hypertension in Egypt. Saudi Journal of Kidney Diseases and Transplantation, 10, 352-356.

[4] Ibrahim, M.M (1997) Hypertension Survey in Developing World. Lesson from the Egyptian National Hypertension Project. Journal of Human Hypertension, 11, 709-726. https://doi.org/10.1038/sj.jhh.1000460

[5] Khalil, H.H. (1996) Hypertension in Elderly Egyptians. Eastern Mediterranean Health Journal, 2, 206-210.

[6] Ibrahim, M.M., Rizk, H., Appel, L.J., et al. (1995) Hypertension Prevelance, Awareness, Treatment, and Control in Egypt. Results from the Egyptian National Hypertension Project. NPH Investigative Team. Hypertension, 26, 886-890. https://doi.org/10.1161/01.HYP.26.6.886

[7] Shimbo, D., Muntner, P., Mann, D., Barr, R.G., Tang, W., Post, W., et al. (2011) Association of Left Ventricular Hypertrophy with Incident Hypertension: The Multi-Ethnic Study of Atherosclerosis. American Journal of Epidemiology, 173, 898-905. https://doi.org/10.1093/aje/kwq509

[8] Nakatani, S. (2011) Left Ventricular Rotation and Twist: Why Should We Learn. Journal of Cardiovascular Ultrasound, 19, 1-6. https://doi.org/10.4250/jcu.2011.19.1.1

[9] Gorman3rd, J.H., Gupta, K.B., Streicher, J.T., Gorman, R.C., Jackson, B.M., Ratcliffe, M.B., Bogen, D.K. and Edmunds Jr, L.H. (1996) Dynamic Three-Dimensional 
Imaging of the Mitral Valve and Left Ventricle by Rapid Sonomicrometry Array Localization. The Journal of Thoracic and Cardiovascular Surgery, 112, 712-726. https://doi.org/10.1016/S0022-5223(96)70056-9

[10] Bansa, M. and Kasliwal, R.R. (2013) How Do I Do It? Speckle Tracking Echocardiography. Indian Heart Journal, 65, 117-123.

https://doi.org/10.1016/j.ihj.2012.12.004

[11] Sengupta, P.P., Krishnamoorthy, V.K. and Korinek, J. (2007) Left Ventricular Form and Function Revisited: Applied Translational Science to Cardiovascular Ultrasound Imaging. Journal of the American Society of Echocardiography, 20, 539-551. https://doi.org/10.1016/j.echo.2006.10.013

[12] Mor-Avi, V., Lang, R.M. and Badano, L.P. (2011) Current and Evolving Echocardiographic Techniques for the Quantitative Evaluation of Cardiac Mechanics: ASE/EAE Consensus Statement on Methodology and Indications Endorsed by the Japanese Society of Echocardiography. Journal of the American Society of Echocardiography, 24, 277-313. https://doi.org/10.1016/j.echo.2011.01.015

[13] Blessberger, H. and Binder, T. (2010) Non-Invasive Imaging: Two Dimensional Speckle Tracking Echocardiography: Basic Principles. Heart, 96, 716-722. https://doi.org/10.1136/hrt.2007.141002

[14] Dalen, H., Thorstensen, A. and Aase, S.A. (2010) Segmental and Global Longitudinal Strain and Strain Rate Based on Echocardiography of 1266 Healthy Individuals: The HUNT Study in Norway. European Journal of Echocardiography, 11, 176-183.

[15] Sengupta, P.P., Tajik, A.J., Chandrasekaran, K. and Khandheria, B.K. (2008) Twist Mechanics of the Left Ventricle: Principles and Application. JACC: Cardiovascular Imaging, 1, 366-376. https://doi.org/10.1016/j.jcmg.2008.02.006

[16] Elgendy, M.S. and Elgendy, A.M. (2013) Essentials of Cardiovascular Medicine, Arterial Hypertension. ESC Guidelines, No. 23, 630-645.

[17] Patel, P. and Macerollo, A. (2010) Diabetes Mellitus: Diagnosis and Screening. American Family Physician, 81, 863-870.

[18] Lang, R.M., Bierig, M., Devereux, R.B., et al. (2005) Recommendations for Chamber Quantification: A Report from the American Society of Echocardiography's Guidelines and Chamber Quantification Writing Group, Developed in Conjunction with the European Association of Echocardiography, a Branch of the European Society of Cardiology. Journal of the American Society of Echocardiography, 18, 1440-1463. https://doi.org/10.1016/j.echo.2005.10.005

[19] Devereux, R.B., Alonso, D.R., Lutas, E.M., Gottlieb, G.J., Campo, E., Sachs, I., et al: (1986) Echocardiographic Assessment of Left Ventricular Hypertrophy: Comparison to Necropsy Findings. American Journal of Cardiology, 57, 450-458. https://doi.org/10.1016/0002-9149(86)90771-X

[20] Li, L., Shigematsu, Y., Hamada, M. and Hiwada, K. (2001) Relative Wall Thickness Is an Independent Predictor of Left Ventricular Systolic and Diastolic Dysfunctions in Essential Hypertension. Hypertension Research, 24, 493-499. https://doi.org/10.1291/hypres.24.493

[21] Chobanian, A.V., Bakris, G.L., Black, H.R., et al. (2003) Seventh Report of the Joint National Committee on Prevention, Detection, Evaluation, Investigations, and Treatment of High Blood Pressure. Hypertension, 429, 1206-1252. https://doi.org/10.1161/01.HYP.0000107251.49515.c2

[22] Marwick, T.H., et al. (2015) Recommendations on the Use of Echocardiography in Adult Hypertension. JASE, 28, 1-12.

[23] Bradshaw, A.D., Baicu, C.F., Rentz, T.J., et al. (2010) Age-Dependent Alterations in 
Fibrillar Collagen Content and Myocardial Diastolic Function: Role of SPARC in Post-Synthetic Procollagen Processing. American Journal of Physiology-Heart and Circulatory Physiology, 298, 614-622. https://doi.org/10.1152/ajpheart.00474.2009

[24] Buckberg, G., Hoffman, J.I., Nanda, N.C., et al. (2011) Ventricular Torsion and Untwisting: Further Insights into Mechanics and Timing Interdependence: A Viewpoint. Echocardiography, 28, 782-804. https://doi.org/10.1111/j.1540-8175.2011.01448.x

[25] Cameli, M., Lisi, M., Righini, F.M., et al. (2013) Left Ventricular Remodeling and Torsion Dynamics in Hypertensive Patients. The International Journal of Cardiovascular Imaging, 29, 79-86. https://doi.org/10.1007/s10554-012-0054-0

[26] Han, W., Xie, M., Wang, X., et al. (2008) Assessment of Left Ventricular Global Twist in Essential Hypertensive Heart by Speckle Tracking Imaging. Journal of Huazhong University of Science and Technology, 28, 114-117.

[27] De Simone, G., Devereux, R.B., Roman, M.J., Ganau, A., Saba, P.S., Alderman, M.H. and Laragh, J.H. (1994) Assessment of Left Ventricular Function by the Midwall Fractional Shortening/End-Systolic Stress Relation in Human Hypertension. Journal of the American College of Cardiology, 23, 1444-1451. https://doi.org/10.1016/0735-1097(94)90390-5

[28] Soufi Taleb Bendiab, N., et al. (2017) Factors Associated with Global Longitudinal Strain Decline in Hypertensive Patients with Normal Left Ventricular Ejection Fraction. European Journal of Preventive Cardiology, 24, 1463-1472. https://doi.org/10.1177/2047487317721644

[29] Abdou, W., Abdallah, I.H., Kamal, M., et al. (2014) Evaluation of Left Ventricular Performance in Hypertensive Patients by Speckle Tracking Echocardiography: Correlation with Brain Natriuretic Peptide. The Egyptian Heart Journal, 66, 299-308. https://doi.org/10.1016/j.ehj.2014.08.002

[30] Gncalvesa, S., Cortez-Diasb, N., Nunesa, A., et al. (2014) Left Ventricular Systolic Dysfunction Detected in Hypertensive Patients with Preserved Ejection Fraction. Revista Portuguesa de Cardiologia, 33, 27-37.

[31] Imbalzano, E., Zito, C., Carerj, S., et al. (2014) Left Ventricular Function in Hypertension: New Insight by Speckle Tracking Echocardiography. Echocardiography, 28, 649-657. https://doi.org/10.1111/j.1540-8175.2011.01410.x

[32] Rosa, E.C., Moysés, V.A., Rivera, I., et al. (2002) Left Ventricular Diastolic Function in Essential Hypertensive Patients. Influence of Age and Left Ventricular Geometry. Arquivos Brasileiros de Cardiologia, 78, 472-477.

[33] Haung, J., Yan, Z.N., Ni, X.D., et al. (2016) Left Ventricular Longitudinal Rotation Changes in Primary Hypertensive Patients with Normal Left Ventricular Ejection Fraction Detected by Two-Dimensional Speckle Tracking Imaging. Journal of Human Hypertension, 30, 30-34. https://doi.org/10.1038/jhh.2015.25

[34] Wang, F., Feng, X., Chen, B., et al. (2011) Evaluation of Left Ventricular Diastolic Function by Tissue Doppler Echocardiography in Essential Hypertension. Heart, 97, A196.

[35] Knežević, B., Musić, L., Bulatović, N. and Nenezić, A. (2015) Systolic and Diastolic Function in Hypertension. Journal of Hypertension Research, 1, 88-94. 